\title{
Energy Optimization in Wireless Sensor Network Using Sleep Mode Transceiver
}

\author{
C.Umamaheswari ${ }^{1}$, M.E, R.V.Viswanathan ${ }^{2}$, M.E, R.Sangeetha ${ }^{2}$, M.E, \\ ${ }^{1}$ Assistant Professor / IT Department, Vivekanandha College of Engineering For Women \\ ${ }^{2}$ Assistant Professor / IT Department, Vivekanandha College of Engineering For Women \\ ${ }^{2}$ Assistant Professor / IT Department, Vivekanandha College of Engineering For Women
}

\begin{abstract}
Energy efficiency is a central challenge in sensor networks, and the radio is a major contributor to overall energy node consumption. These Wireless Sensor Networks have severe resource constrains and energy conservation is very essential. The aim of this project is to reduce the energy consumption in wireless sensor networks. This paper proposes adaptive radio low-power sleep modes based on current traffic conditions in the network. It provides an analytical model to conduct a comparative study of different MAC protocols (BMAC, TMAC, SMAC, DMAC) suitable for reduction of energy consumption in wireless environment. This technique exposes the energy trade-offs of different MAC protocols. It first introduces a comprehensive node energy model, which includes energy components for radio switching, transmission, reception, listening, and sleeping for determining the optimal sleep mode and MAC protocol to use for given traffic scenarios. The model is then used for evaluating the energy-related performance of our recently proposed RFID Impulse protocol enhanced with adaptive low-power modes, and comparing it against BMAC under varying data rates. The comparative analysis confirms that RFID Impulse with adaptive low power modes provides lower energy consumption than the BMAC and DMAC in low traffic scenario. The evaluation also yields the optimal settings of low-power modes on the basis of data rates for node platform, and provides guidelines and a simple algorithm for the selection of appropriate MAC protocol, low-power mode, traffic requirements of a sensor network application.

Index Terms: RFID, wake-up radio, sleep mode, adaptive, energy efficiency, MAC protocols, routing protocols, energy model, sensor networks.
\end{abstract}

\section{Introduction:}

Energy efficiency is a central challenge in sensor networks, as battery replacement is costly and often difficult in inaccessible deployment regions. Several efforts have addressed energy efficiency in sensor networks, through the design of energy saving MAC protocols, such as duty cycling protocols [7] or low-power wake-up radio protocols [12], and routing protocols, such as [11].

Radio energy consumption is a major component contributing to the overall energy consumption at each node. Current MAC protocols put the radio in sleep mode while there is no data to send or receive, in order to minimize energy consumption. Although most radios for sensor networks support multiple sleep modes, the radio sleep mode in current MAC protocols is static. Choosing a static low-power mode involves an energy and delay tradeoff.

The deepest sleep mode, which turns off the oscillator and voltage regulator, provides the lowest current draw of all low-power modes. However, it also involves the highest energy cost and the longest latency for switching the radio back to active mode. In contrast, the lightest sleep mode provides a transition to active mode that is quick and energy inexpensive, but it has a higher current draw. In a low traffic scenario, it is better to use the deep sleep mode as nodes spend more time sleeping than switching back and forth between sleep mode and active mode. In a high traffic scenario, a lighter sleep mode is more suitable as the cost of switching the radio frequently into deep sleep mode would exceed the energy saving of the deep sleep mode's low current draw.

To address this trade-off, this paper proposes adaptive radio power modes that dynamically change according to current traffic conditions in the network. To demonstrate the benefits of adaptive sleep modes, we incorporate them into our recently proposed RFID Impulse mechanism [15], [2], and compare its performance against the popular BMAC [7] protocol. The performance evaluation of proposed protocols generally considers the radio energy consumption, including receiving, transmitting, listening, and sleeping energy consumption components, but it disregards the switching energy component [16] that is appreciable for any protocol that switches nodes between active and sleep modes in low traffic conditions.

In order to determine how to adapt low-power modes in RFID Impulse and to compare the MAC protocols, this paper presents a sensor node energy consumption model that includes switching energy. The model enhances existing models [7], is generalizable to any MAC protocol, and serves as the basis for evaluating the energy consumption of sleep mode configurations for given traffic loads and for determining the 
optimal protocol/sleep mode configuration and for comparing the protocols through a custom-built Matlab simulation model [2].

The comparison of the protocols under different traffic loads yields guidelines for selecting appropriate MAC protocols for specific traffic requirements of an application. We also determine the optimal radio low power mode within RFID Impulse as the data rate varies. Proposal of adaptive radio low-power sleep modes within our previously proposed RFID Impulse protocol that can dynamically change based on network or node traffic.

Introduction of an energy model that considers radio energy consumption, including transmission, reception, listening, sleeping, and switching energy components, as an enabler for comparing protocols across node platforms that use different processor boards. Presentation of a simple algorithm based on the energy model for selecting the optimal protocol and sleep mode configuration for a given traffic load.

Energy efficiency evaluation of BMAC, and RFID Impulse. The evaluation considers the dependence of energy efficiency and optimal power mode on data rate. Provision of guidelines based on the evaluation results for MAC protocol, power mode, selection according to the expected traffic requirements of the target application.

\section{Mac Protocols}

This section presents the three protocols under consideration separately: BMAC, DMAC and RFID Impulse.

\subsection{BMAC}

BMAC [7] is an asynchronous and lightweight sensor network MAC protocol that aims at providing versatile medium access while keeping the MAC functionality as simple as possible. As an asynchronous protocol, BMAC eliminates the communication and processing overhead for scheduling and synchronization, which reduces energy consumption.

BMAC enables each node to wake up periodically to check for channel activity. The wake-up period is referred to as the check interval. BMAC defines eight check intervals, and each check interval corresponds to one of BMAC's eight listening modes. To ensure that all packets are heard by neighboring nodes, packets are sent with a preamble whose reception time is longer than the check interval. BMAC, therefore, defines eight different preamble lengths referred to as transmit modes. Although several optimizations have improved over BMAC.

\subsection{DMAC}

The Data-Gathering Medium Access Control (DMAC) is a schedule based MAC protocol which has been designed and optimized for tree based data gathering (converge cast communication) in wireless sensor network. The main objective of this MAC protocol is to achieve low latency and still maintaining the energy efficiency. In this protocol the time is divided in small slots and runs carrier sensing multiple access (CSMA) with acknowledgement within each slot to transmit/receive one packet. The sensor node periodically executes the basic sequence of ' 1 ' transmit, ' 1 ' receive and ' $n$ ' sleep slots.

\subsection{RFIDIMPULSE}

RFID Impulse is a very low-power radio wake-up scheme for sensor networks that relies on off-theshelf RFID readers and tags. The Basis functionality of RFID Impulse is that all network nodes turn off their radios, including the voltage regulator and the oscillator, as long as they have no packets to send or receive and sends a short acknowledgment message through the standard radio to the sender, indicating that the RFID wakeup was successful. Upon receiving the short acknowledgment from the receiver, the sender commences the transmission. Once the sender completes all its packet transmissions, both sender and receiver again turn off their radios If a sender fails to receive an acknowledgment from the If a sender fails to receive an acknowledgment from the receiver in response to an RFID wake-up signal, the sender assumes that the receiver tag did not detect the signal or that the signal level is too low to activate the receiver tag. The sender then transmits the RFID wake-up signal again, with a maximum of three retries. If the receiver acknowledges receipt of one of the RFID wake-up signals, then the sender proceeds with the transmission. Otherwise, the sender ceases its attempts to use RFID wake-up signals to this receiver.

\subsubsection{Trade-Offs}

To address the energy trade-off, RFID Impulse supports traffic-based selection of low-power radio modes. As a general rule, when the traffic load is high in a particular region of the network, nodes use lighter sleep modes as they have to wake up frequently to send and receive packets. It is not worthwhile for nodes to go 
into deeper sleep modes due to the higher latency and switching energy involved in frequent wake-up transitions.

When the traffic load is low in a particular region of the network, switching between sleep and active states is less frequent, so nodes use deeper sleep modes that provide the highest energy savings. Determining quantitative thresholds for optimal sleep mode selection demands an energy model that captures all components contributing to energy consumption at sensor nodes.

\section{Analytical Model}

In order to model the energy consumption of the three MAC protocols, this section considers all the energy components that contribute to the overall energy consumption at a node including the radio.

Consider a convergecast application where all nodes sample their sensor periodically and send the data toward the base station. The discussion here focuses on energy consumption during a single sampling period.

\subsection{Listening Energy}

We define the listening energy as the radio energy consumption when the radio is active but not receiving or sending any packets. Protocols that are based on low-power listening, such as BMAC [6], have the following listening energy:

$$
E_{l}^{l p l}=\frac{S}{C K} * T_{C H} * I_{\text {listen }} * V
$$

where $\mathrm{S}$ is the sampling period, $\mathrm{CK}$ is the check interval, $T_{C H}$ is the time during which the node $I_{\text {listen }}$ isawake every cycle, and is the current draw of the radio in listening mode.

\subsection{Switching Energy}

The switching energy component [15] is the energy consumed for switching the radio state between states, including normal, power down, and idle modes. The following equation determines the energy consumed for switching the radio from sleep mode $\alpha$ to active mode:

$$
E_{\text {switch }}^{\alpha}=\frac{\left(I_{\text {active }}-I_{\propto)} * T_{\propto} * V\right.}{2}
$$

Where $I_{\text {active }}$ is the current draw of the radio in active mode, $I_{\alpha}$ is the current draw of the radio in sleep mode $\alpha$, and $T_{\alpha}$ is the time required for the radio to go from sleep mode $\alpha$ to active mode. Equation assumes that the energy variation varies linearly while switching between sleep mode $\alpha$ and active mode. While the energy consumption due to switching can follow a nonlinear pattern, the linear approximation is reasonable for differentiating between the high cost of switching from a deep sleep mode and the low cost of switching from a light sleep mode.

\subsection{Transmission Energy}

The transmission energy component refers to the energy consumed for transmitting packets and their associated control overhead on the radio. During any time period, the transmission energy is expressed as

$$
E_{t}=P_{\text {sent }} * P_{\text {lenght }} * T_{B} * I_{t} * V
$$

where $P_{\text {lenght }}$ is the length of a packet in bytes, It is the current draw of the radio while in transmit mode, and $T_{B}$ is the time for sending 1 byte over the radio.

\subsection{Receiving Energy}

The reception energy component refers to the energy consumed while receiving packets and their associated control overhead on the radio. During any time period, the reception energy is expressed as

$$
E_{t}=P_{\text {recv }} * P_{\text {lenght }} * T_{B} * I_{r} * V
$$

where $I_{r}$ is the current draw of the radio while receiving.

\subsection{Sleeping Energy}

The sleeping energy component is simply the energy consumption, while the radio is in low-power mode. The following equation computes the sleeping energy for a node that goes into sleep mode $\alpha$ when it is off:

\subsection{Overall Energy}

$$
E_{\text {sleep }}=T_{r f}^{o f f} * I_{\alpha} * V
$$

The overall energy consumption at each node using protocol P and sleep mode $\alpha$ is simply the sum of all of the above energy components of that node for the given protocol:

$$
E_{p}^{\alpha}=E_{l} * E_{\text {switch }} * E_{t} * E_{r} * E_{\text {sleep }}
$$


The selection of the optimal MAC protocol P and sleep mode $\alpha$ for a given network scenario can thus consider all available MAC protocols and sleep modes, and pick the combination of protocol and sleep mode that yields the lowest energy consumption.

The energy model described in this section provides the basis for evaluating energy performance, protocol trade-offs.

\subsection{Delay Considerations}

To understand the implications of using different low-power radio modes on the end-to-end delay, we consider a simple delay model that considers $T_{\propto}$ for a given sleep mode $\alpha$, the packet transmission time $T_{t}$, the backoff time Tbo, and the queuing delay $T_{q}$. Note that the transmission time $\mathrm{Tt}=\mathrm{T}_{\mathrm{B}}{ }^{*} \mathrm{P}_{\text {lenght }}$ and $\mathrm{Tbo}=\mathrm{T}_{\mathrm{B}} * 54$ in a highly congested scenario for IEEE 802.15.4 radios.

\section{Performance Evaluation}

This section explores the interdependencies among MAC protocols, node platforms, and traffic load in sensor networks. We built our energy model from the previous section into a custom Matlab simulator. The evaluation here considers three MAC protocols: 1) the widely used BMAC protocol, 2) DMAC 3) RFID Impulse.

The first part of this section exposes the energy trade-offs of the three MAC protocols for a low sampling rate multihop scenario and a high sampling rate multihop scenario. The goal of these simulations is to expose the dominant energy components for each protocol on the basis of traffic load

The second part of this section considers the effects of traffic forwarding on energy consumption under four fixed sampling periods. Building on these results, the third part of this section determines the energy consumption of each MAC protocol based on useful data rate and identifies the best performing protocol for each node platform and traffic load. The final part of this section examines the delay implications of adaptive radio sleep modes.

\subsection{Energy Trade-Offs}

First explore the energy trade-offs of the three protocols mentioned above. In this evaluation, we consider a network with a 6-hop binary tree static topology. Although the topology of an actual sensor network can be both irregular and transient according to environmental conditions as well as location, this study serves as a representative case that exposes the energy trade-offs of the three MAC protocols under varying traffic loads. The network is convergecast in nature where all nodes periodically sample their sensors and send the data in a packet toward the base station that is colocated with the root of the tree topology. Packets are forwarded in a multihop fashion until they reach the base station. Each node's hop count from the root in the logical topology determines its forwarding load. Intermediate nodes must forward all packets of their children, while leaf nodes only send their own packets. The first scenario considers the energy trade-offs in a 6-hop binary tree network with a low data rate in which the sampling period $\mathrm{S}$ is set to 100 seconds. Because of the low traffic load in this scenario, RFID Impulse uses the deepest sleep mode.

\section{Results}

This section explores the result of the energy trade-offs between these three MAC protocols (RFID Impulse, BMAC, DMAC) and analyses the performance against the each MAC protocols.

Fig. 1 plots the Power consumption trade-offs between the RFID Impulse, BMAC and DMAC when there is an communication between the nodes.

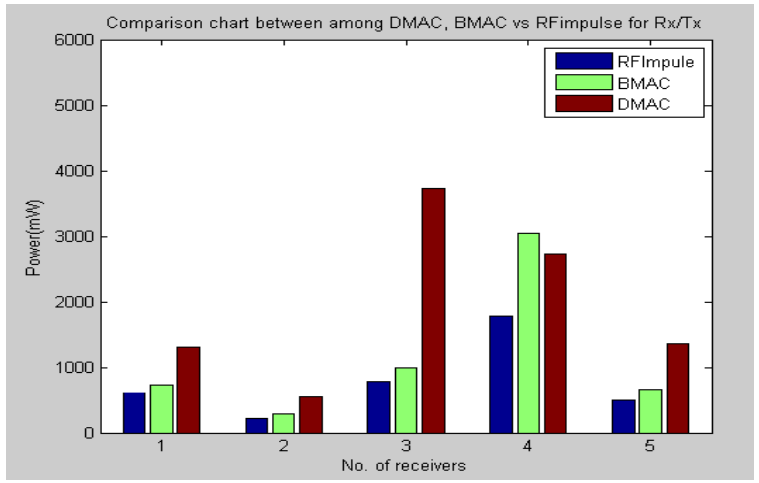

Fig.1.Power consumption trade-offs between the RFID Impulse, BMAC and DMAC for Rx/Tx. 
Fig. 2 plots the Power consumption trade-offs between the RFID Impulse, BMAC and DMAC which shows the energy level for each Mac Protocols when there is switching between the states from sleep mode to awaken mode or awaken mode to sleep mode.

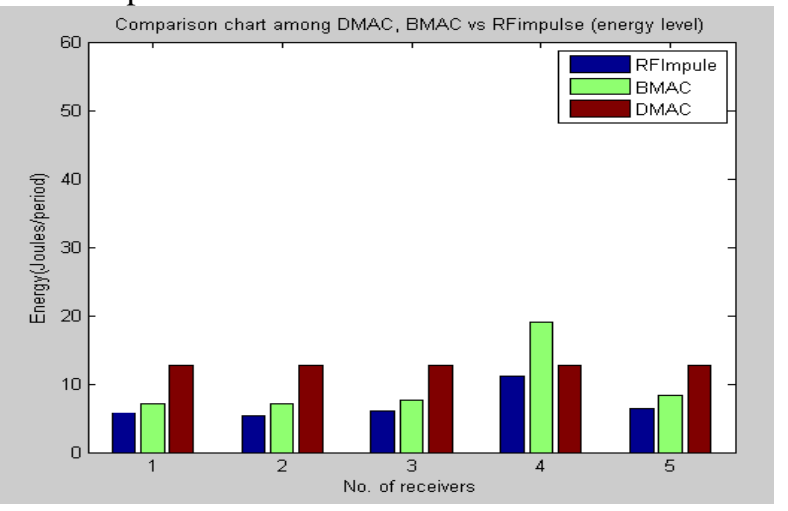

Fig.2.Power consumption trade-offs between the RFID Impulse, BMAC and DMAC which shows the energy level for each Mac Protocols.

\section{Conclusion And Future Work}

This paper has proposed adaptive radio sleep modes as an energy optimization technique for wireless sensor networks. Because nearly all sensor network MAC protocol alternate frequently between sleep and awake states, the frequency of this state switching should dictate the appropriate sleep mode that minimizes energy consumption.

This paper has also provided an analytical model to conduct a comparative study of MAC protocol suitability of BMAC, DMAC, and the newly proposed RFID Impulse Building on the dependence of protocol performance on traffic loads, the paper has also quantitatively explored the application of adaptive low-power radio sleep modes based on the level of data traffic in the network

Our analyses of the delay implications of using adaptive sleep modes have considered uniform delay along the routing tree. In reality, queuing and collision backoff delay components are higher at critical nodes near the base station. This effect is purely dependent on the structure of convergecast topologies and not on the use of adaptive sleep modes. In fact, critical nodes may exhibit higher queuing and collision backoff delays because of the higher traffic rates in the vicinity of these nodes. If these nodes use adaptive sleep modes, they will select the light sleep mode for their operation as a direct consequence of their higher forwarding load which minimizes $T \alpha$, and consequently, reduces the end-to-end delay for the packets these nodes forward.

From comparative analysis confirms that RFID Impulse with adaptive low power modes provides lower energy consumption than the BMAC and DMAC in low traffic scenario. The evaluation also yields the optimal settings of low-power modes on the basis of data rates for node platform, and provides guidelines and a simple algorithm for the selection of appropriate MAC protocol, low-power mode, traffic requirements of a sensor network application.

An interesting direction for future work is to implement RFID Impulse by attaching RFID tags to the external interrupt pin of sensor node MCUs, and then, configuring the radio to trigger the remote tags.

Cross-layer dependencies in sensor networks [19] require consideration of not only energy performance based on the choice of hardware and MAC protocols, but also the delay performance and the choice of routing and scheduling protocols as well.

An interesting direction for future work is to explore the interdependencies and between the choice of node platforms, MAC protocols, and routing and scheduling protocols. Keeping in mind that these dependencies exist, the measurement-based comparative study in this paper will hopefully serve as a guide for designers and researchers in selecting node platforms and MAC protocols that are suitable for the expected traffic requirements in their applications.

\section{References}

[1] Radio Sleep Mode Optimization in Wireless Sensor Networks Raja Jurdak, Member, IEEE, Antonio G. Ruzzelli, and Gregory M.P. O'Hare

[2] R. Jurdak, A.G. Ruzzelli, and G.M.P. O’Hare, “Adaptive Radio Modes in Sensor Networks: How Deep to Sleep?” Proc. IEEE Comm. Soc. Conf. Ad Hoc and Sensor Networks (SECON '08), June 2008.

[3] R. Want, "Enabling Ubiquitous Sensing with RFID", Computer, vol. 37, no. 4, pp. 84-86, Apr. 2004.

[4] Atmel Atmega128,http://www.atmel.com, 2010.

[5] Texas Instruments cc2420 Radio Transceiver, http://focus.ti.com/docs/prod/folders/print/cc2420.html, 2010

[6] V. Shnayder, M. Hempstread, B. Chen, G.W. Allen, and M. Walsh, "Simulating the Power Consumption of Large-Scale Sensor Network Applications", Proc. ACM Int'l Conf. Embedded Networked Sensor Systems (Sensys '04), 2004. 
[7] J. Polastre, J. Hill, and D. Culler, "Versatile Low Power Media Access for Wireless Sensor Networks", Proc. ACM Int'l Conf. Embedded Networked Sensor Systems (Sensys '04), 2004.

[8] R. Jurdak, P. Baldi, and C.V. Lopes, Adaptive Low Power Listening for Wireless Sensor Networks", IEEE Trans. Mobile Computing., vol. 6, no. 8, pp. 988-1004, Aug. 2007.

[9] MicaZ Mote Platform, Crossbow Technologies, http://www.xbow.com/Products/Product_pdf_files/Wireless_pdf/MICAZ_ Datasheet.pdf, 2010.

[10] TelosB Mote Platform, Crossbow Technologies, http://www.xbow.com/Products/Product_pdf_files/Wireless_pdf/ TelosB_Datasheet.pdf, 2010 .

[11] A.G. Ruzzelli, G.M.P O'Hare, and R. Jurdak, "MERLIN: Cross-Layer Integration of MAC and Routing for Low Duty-Cycle Sensor Networks", Ad Hoc Networks J., vol. 6, no. 8, pp. 1238-1257, Feb. 2008.

[12] L. Gu and J.A. Stankovic, "Radio-Triggered Wake-Up Capability for Sensor Networks", Proc. 10th IEEE Real-Time and Embedded Technology and Applications Symp. 2004.

[13] Tag Sense: RFID and Wireless Sensing, http://www.tagsense.com, 2010.

[14] P. Skraba, H. Aghajan, and A. Bahai, "RFID Wake-Up in Event Driven Sensor Networks", technical report, U.C. Berkeley, 2001.

[15] A.G. Ruzzelli, R. Jurdak, and G.M.P. O'Hare, "On the RFID Wake-Up Impulse for Multi-Hop Sensor Networks", Proc. Convergence of RFID and Wireless Sensor Networks and Their Applications (SenseID) Workshop at ACM Int'l Conf. Embedded Networked Sensor Systems (Sensys '07), Nov. 2007.

[16] A.G. Ruzzelli, P. Cotan, G.M.P. O'Hare, R. Tynan, and P.J.M Havinga, "Protocol Assessment Issues in Low Duty Cycle Sensor Networks: The Switching Energy", Proc. IEEE Int'l Conf. Sensor Networks, Ubiquitous, and Trustworthy Computing (SUTC '06), June 2006. WPAN's),IEEE,http://www.ieee802.org/15/pub/TG4.html, 2010.

[18] TagSense, Inc., “ZT-10 Active RFID Tag,” http://www.tagsense.com/ingles/products/products/ZT-10-tag-v4-5.pdf, 2010.

[19] R. Jurdak, Wireless Ad Hoc and Sensor Networks: A Cross-Layer Design Perspecitve. Springer-Verlag, Jan. 2007. 